\title{
Characteristics of Treated Asthmatics Experiencing Exacerbations in a US Database: A Retrospective Cohort Study
}

\author{
Carlyne M Averell' \\ David Hinds ${ }^{2}$ \\ Jolyon Fairburn-Beech (iD) ${ }^{3}$ \\ Benjamin Wu' \\ Robson Lima ${ }^{4}$ \\ 'US Value Evidence \& Outcomes, GSK, \\ Research Triangle Park, NC, USA; ${ }^{2}$ Real \\ World Evidence \& Epidemiology, GSK, \\ Collegeville, PA, USA; ${ }^{3}$ Real World \\ Evidence \& Epidemiology, GSK, Stockley \\ Park, UK; ${ }^{4}$ US Medical Affairs, GSK, \\ Research Triangle Park, NC, USA
}

Rationale: The National Heart, Lung, and Blood Institute (NHLBI) recommend a stepwise approach to asthma management, with the goals of maintaining asthma control and reducing exacerbations. Although asthma treatments reduce the frequency of exacerbations, they still occur. We aimed to characterize the treated United States of America (US) adult asthma population, including those experiencing exacerbations, in terms of socio-demographics, clinical characteristics, and healthcare resource utilization (HRU).

Patients and Methods: A retrospective cohort of asthma patients aged $\geq 18$ years on 01 January 2014 with $\geq 1$ ICD-9 asthma code (493.xx) enrolled in a US healthcare claims database during 2013-2014. Patients who had $\geq 2$ asthma medication dispensings during 2013 (baseline), including $\geq 1$ in the 90 -day period before index date, were classified according to NHLBI step. Patients with chronic obstructive pulmonary disease, cystic fibrosis, or lung cancer diagnoses were excluded. Demographics, comorbidities, clinical characteristics, and HRU were described during baseline. Exacerbations and HRU were described during 2014 (follow-up period).

Results: In total, 72,156 patients were included; 10,590 (14.7\%) had $\geq 1$ exacerbation during follow-up. Approximately $44 \%$ of patients were classified as NHLBI Steps $1-2,41 \%$ as Steps 3-4, and $11 \%$ as Steps 5-6. Exacerbation frequency increased with step (Steps 1, 2, and 3: $12-14 \%$; Steps 4, 5, and 6: 16-26\%). Compared with the overall population during baseline, patients with an exacerbation had similar demographics, but differences were observed for comorbid allergic rhinitis ( $46.4 \%$ vs $40.1 \%$, respectively), blood eosinophil counts $\geq 300$ cells $/ \mu \mathrm{L}$ ( $45.5 \%$ vs $39.6 \%$, respectively), and asthma-related healthcare encounters $(62.9 \%$ vs $52.4 \%$, respectively). Overall, asthma-related HRU during follow-up increased with NHLBI step.

Conclusion: Exacerbations were observed among patients classified at each NHLBI step and were more frequent with increasing step. Exacerbations and asthma-related HRU highlight the continued unmet need in the treated US asthma population.

Keywords: asthma, clinical traits, real-world, administrative claims, healthcare costs, healthcare resource utilization

\section{Introduction}

Asthma affects approximately 300-350 million people worldwide and over 25 million individuals in the United States of America (US). ${ }^{1,2}$ Patients with asthma often experience episodes of worsening symptoms, known as exacerbations, which often vary in severity and frequency. ${ }^{3}$ A significant proportion of healthcare costs and morbidity derive from these acute deteriorations. For example, in the US alone, around
Correspondence: Carlyne M Averell US Value Evidence \& Outcomes, GlaxoSmithKline plc., Research Triangle Park, NC, 27709-3398, USA

$\mathrm{Tel}+19193159835$

Email Carlyne.M.Averell@gsk.com 
15 million outpatient visits, 2 million emergency department (ED) visits, and 500,000 hospitalizations occur each year during the management of acute asthma. ${ }^{4}$ The burden associated with these asthma-related exacerbations remains significant ${ }^{5-8}$ despite the refinement of asthma management guidelines, development of new treatments, and increases in medication use.

The clinical severity of asthma is indicated by symptoms including sleep disturbance, limitation of daily activity, lung function impairment, and the use of rescue medications. ${ }^{9,10}$ When patients have uncontrolled asthma, they experience significantly impaired quality of life and increased asthma-related healthcare costs. ${ }^{9,11}$ In particular, exacerbations requiring oral corticosteroid (OCS) treatment and/or ED visits or hospitalization have been reported as posing the most significant burden to healthcare systems. $^{12-15}$

Effective treatments to enhance asthma control have been developed, including inhaled corticosteroids (ICS) and ICS/long-acting beta-agonist combinations. However, despite these advances in asthma management, exacerbations continue to occur. ${ }^{16-19}$

Clinical guidelines aiming to improve long-term asthma control (including reduction of exacerbation risk) include those from the Global Initiative for Asthma (GINA) ${ }^{9}$ and the National Heart, Lung, and Blood Institute (NHLBI). ${ }^{20,21}$ Previous studies have described exacerbation frequencies and clinical characteristics in patients from the United Kingdom and US using the GINA guidelines' step categorization of asthma treatment; ${ }^{22}$ however, no study has yet reported exacerbations according to the steps described in the NHLBI guidelines. $^{21}$

We aimed to characterize the treated adult asthma population in the US, including those experiencing exacerbations, using the NHLBI guidelines' treatment step categories, to provide insights regarding potential unmet needs of US asthma patients.

\section{Patients and Methods Objectives}

The objectives of this study were two-fold. First, to characterize the US adult asthma population treated with asthma controller medication(s) and/or rescue medication according to the NHLBI stepwise treatment categories, thus providing a proxy of asthma severity (Figure 1). Second, to characterize the treated US adult asthma population who experienced exacerbations during 2014 according to the NHLBI stepwise treatment categories. In order to describe these populations, the following variables were assessed: baseline demographics and clinical characteristics; comorbid conditions; asthma treatments (including adherence); total and asthma-related healthcare resource utilization (HRU) and costs; and asthma exacerbations (including frequency, type, and seasonality).

\section{Study Design}

We conducted a retrospective cohort study of adult patients in 2013-2014 using a large US private health insurance claims database: Optum's de-identified CLINFORMATICS Data Mart Database (hereafter referred to as the Optum database). The index date was 01 January 2014, with the respiratory treatment(s) in the 90 days before the index date used to classify patients according to the six NHLBI steps. Patient demographics, comorbidities, exacerbations, and HRU and costs were described in the 12 months before the index date and termed as the baseline period (01 January 2013 to 31 December 2013). The 12-month period after the index date was termed the follow-up period (01 January 2014 to 31 December 2014) and was used to describe the occurrence of asthma exacerbations, treatment changes (discontinuation, augmentation), adherence to treatment, HRU, and costs by NHLBI step.

\section{Study Population}

The study population included patients with treated asthma who were identified in the Optum database. Inclusion criteria for the study were: continuous enrollment in the database between 01 January 2013 and 31 December 2014; at least 18 years of age at the index date; at least one diagnosis code for asthma (International Classification of Diseases, 9th revision [ICD-9] 493.xx); and at least two prescriptions for an asthma medication (rescue or maintenance medication) in the baseline period. This included at least one dispensing in the 90 days prior to the index date, which was used to classify the patients according to NHLBI steps. Patients were excluded from the study if they had any diagnosis of chronic obstructive pulmonary disease (ICD-9: 491.xx, 492.xx, 496.xx), cystic fibrosis (ICD-9: 277.0x), acute respiratory failure (ICD-9: 518.81), or lung cancer (ICD-9: 162.xx) in their medical claims records. The subset of adult asthma patients who experienced an exacerbation during the follow-up period was described and characterized similarly to the overall population. 
Intermittent Asthma
Persistent Asthma: Daily Medication

Consult with asthma specialist if Step 4 care or higher is required Consider consultation at Step 3

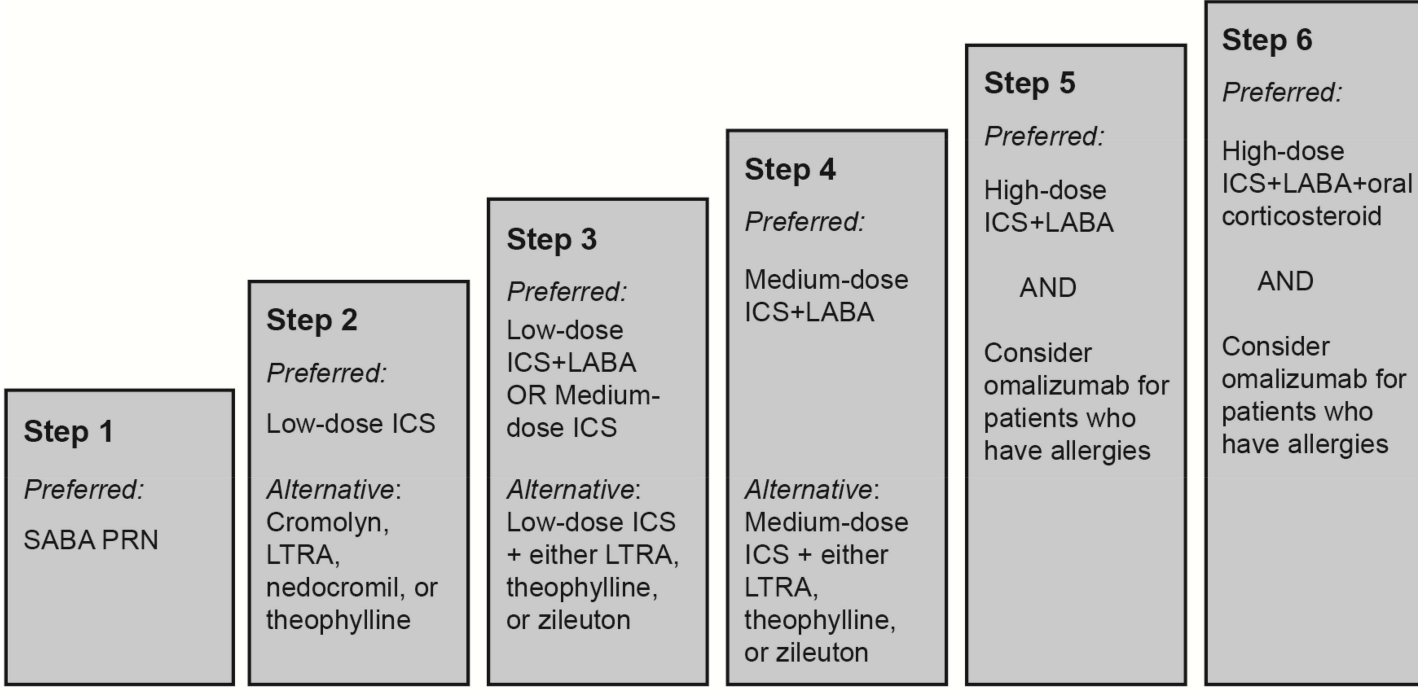

Each step: Patient education, environmental control, and management of comorbidities

Steps 2-4: Consider subcutaneous allergen immunotherapy for patients who have allergic asthma (see notes)

\section{Quick-Relief Medication for All Patients}

- $\quad$ SABA as needed for symptoms. Intensity of treatment depends on severity of symptoms: up to 3 treatments at 20-minute intervals as needed. Short course of oral systemic corticosteroids may be needed

- Use of SABA >2 days a week for symptom relief (not prevention of EIB) generally indicates inadequate control and need to step up treatment

Figure I NHLBI step diagram - illustrates the step-based categorization based on patients' treatment recommended by NHLBI.

Note: Adapted from National Heart, Lung, and Blood Institute (NHLBI); National Institutes of Health; U.S. Department of Health and Human Services. Expert panel report 3: guidelines for the diagnosis and management of asthma. Available from: https://www.nhlbi.nih.gov/health-topics/guidelines-for-diagnosis-management-of-asthma. ${ }^{21}$

Abbreviations: EIB, exercise-induced bronchoconstriction; ICS, inhaled corticosteroid; LABA, long-acting beta agonists; LTRA, leukotriene receptor antagonist; NHLBI, National Heart, Lung, and Blood Institute; PRN, as required; SABA, short-acting beta agonist.

\section{Study Outcomes}

\section{Baseline Patient Demographics and Clinical Characteristics}

Baseline patient characteristics were measured during the 12-month period up to, but excluding, the index date. These included: demographics; atopy status; comorbid conditions; respiratory medications; and eosinophil count level (among those who had eosinophil data available). Atopy status was inferred by the presence of a prior medical claim for allergic rhinitis (ICD-9 of 477.xx) during the baseline period.

\section{NHLBI Asthma Treatment Steps}

The stepwise approach to asthma management recommended by the $\mathrm{NHLBI}^{21}$ was used to assign each treated patient to a corresponding step. These steps are used as a proxy for disease severity, whereby patients are stepped up to the next management level if they are symptomatic despite current treatment. Patients were therefore assigned to each NHLBI step depending on their level of treatment. The NHLBI steps are described in further detail in Figure 1. Patients with a treatment combination not corresponding to one of the steps were considered unclassifiable.

Assignment of NHLBI step was based on asthma medication(s) dispensed closest to the index date along with other asthma medications dispensed during the 90-day period immediately preceding the index date (ie, 01 October 2013-31 December 2013). Days' supply was assumed as 30 days in the case of missing day 
supply value. When assigning treatment steps, we also examined medications that were dispensed prior to the 90-day period if these were still being taken by the patient during the 90-day period (eg, a 30-day supply of an ICS dispensed the day before the 90-day period would be considered in NHLBI step assignment).

\section{Asthma Exacerbations}

Asthma exacerbations were defined as either: 1) an asthma-related ED visit or asthma-related hospital admission, or 2) any OCS use of $\geq 20 \mathrm{mg}$ prednisone (average daily dose) or equivalent lasting at least 3 days (but no more than 28 days) with an asthma medical code recorded within \pm 2 weeks (eg, presence of ICD-9 code 493.x).

ED visits or hospital admissions were determined to be asthma-related by the presence of a primary diagnosis of asthma (ICD-9 code 493.x) on the medical claim. All OCS use other than that described above to define an exacerbation was considered maintenance therapy or non-asthma related. For exacerbations to be considered independent episodes, at least 7 days must have elapsed from the end date of the OCS script, date of ED visit, or hospital discharge date. The seasonality of exacerbations was described based upon the season in which an exacerbation occurred.

\section{Healthcare Resource Utilization}

All-cause and asthma-related HRU were examined during the baseline and 12-month follow-up periods. All-cause HRU was captured as the number of outpatient visits, ED visits, or inpatient admissions for any reason. Asthmarelated HRU (defined as associated with a primary diagnosis code for asthma) was captured as the number of outpatient visits, ED visits, and inpatient admissions.

\section{Healthcare Costs}

All-cause and asthma-related healthcare costs were calculated as total costs, medical costs, and pharmacy costs. These costs were adjusted to 2016 US dollars.

\section{Medication Adherence and Treatment Patterns}

Medication adherence in patients with asthma was evaluated using the proportion of days covered (PDC) by maintenance asthma medication (any ICS, ICS/LABA, leukotriene receptor antagonist, or maintenance OCS), as well as the asthma medication ratio (AMR). ${ }^{23,24}$ The AMR measures the units of maintenance medications dispensed divided by the sum of maintenance and short-acting beta agonist (SABA) medications dispensed. Mean AMR and the percentage of patients with AMR $\geq 0.50$ were calculated during the follow-up period and medication adherence was estimated for patients in each NHLBI step.

Other treatment patterns assessed in the follow-up period included discontinuation of maintenance therapy (defined as no prescription of any maintenance medication after a gap of 60 days after the end of the maintenance medication supply by 31 December 2014), stepping up of therapy (as defined by NHLBI steps), and the number of SABA canisters dispensed.

\section{Data Analysis}

All analyses were descriptive; no statistical analysis was conducted to assess differences between groups. Sample selection and the creation of analytic variables was performed using the Instant Health Data platform (BHE, Boston, MA, US). Descriptive analysis was undertaken with $\mathrm{R}$ version 3.2.1 ( $\mathrm{R}$ Foundation for Statistical Computing, Vienna, Austria).

\section{Results}

\section{Baseline Demographics and Clinical Characteristics}

In total, 72,156 patients met the study inclusion criteria (Table 1; Figure 2). In this overall cohort, patients had a mean age of 49.9 years (standard deviation [SD] $16.17)$ and the majority were female $(64.0 \%)$. During the 12-month baseline period, 15,701 patients $(21.8 \%)$ had at least one exacerbation and 3442 (4.8\%) had a history of at least two exacerbations. The majority of asthma exacerbations among patients in the baseline period were OCS-defined (19.0\%) versus ED/hospital admission (3.8\%). Of the $15,349(21.3 \%)$ patients with an eosinophil value, 6074 patients $(39.6 \%)$ had an eosinophil count $\geq 300$ cells $/ \mu \mathrm{L}$. The percentage of patients with one or more asthma-related healthcare encounter at baseline was $52.4 \%$ for outpatient visits, $3.7 \%$ for ED visits, and $0.8 \%$ for hospitalizations (Table 2).

Among the overall cohort, 10,590 patients (14.7\%) had at least one exacerbation during the follow-up period. This exacerbating cohort was comparable to the overall cohort (mean age 49.0 years, SD 15.4) (Supplementary Table 1). Higher proportions of these patients had blood eosinophil counts $\geq 300$ cells $/ \mu \mathrm{L}$ ( $45.5 \%$ among 2122 patients with blood eosinophil measurements) and one or more asthma- 


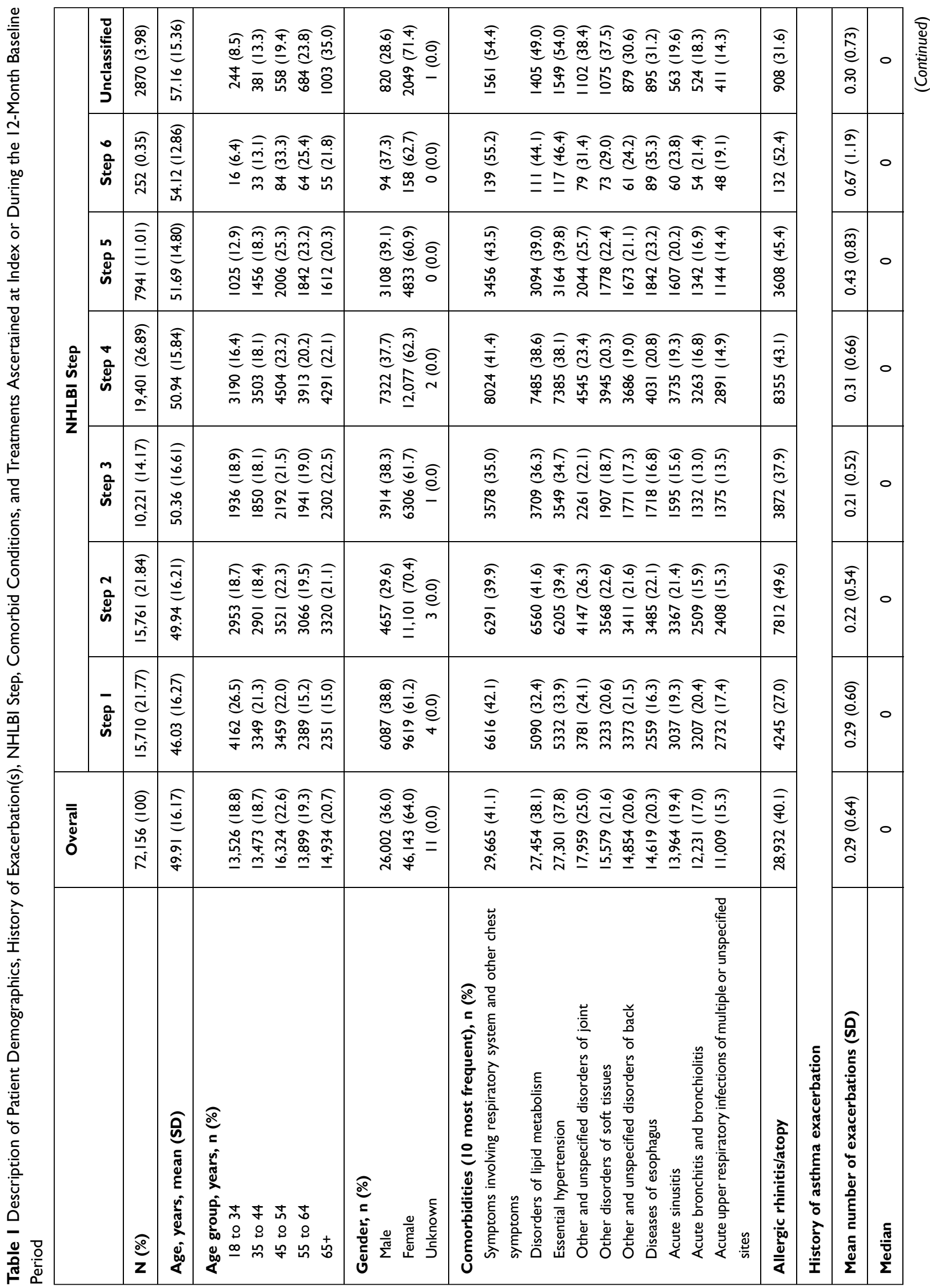




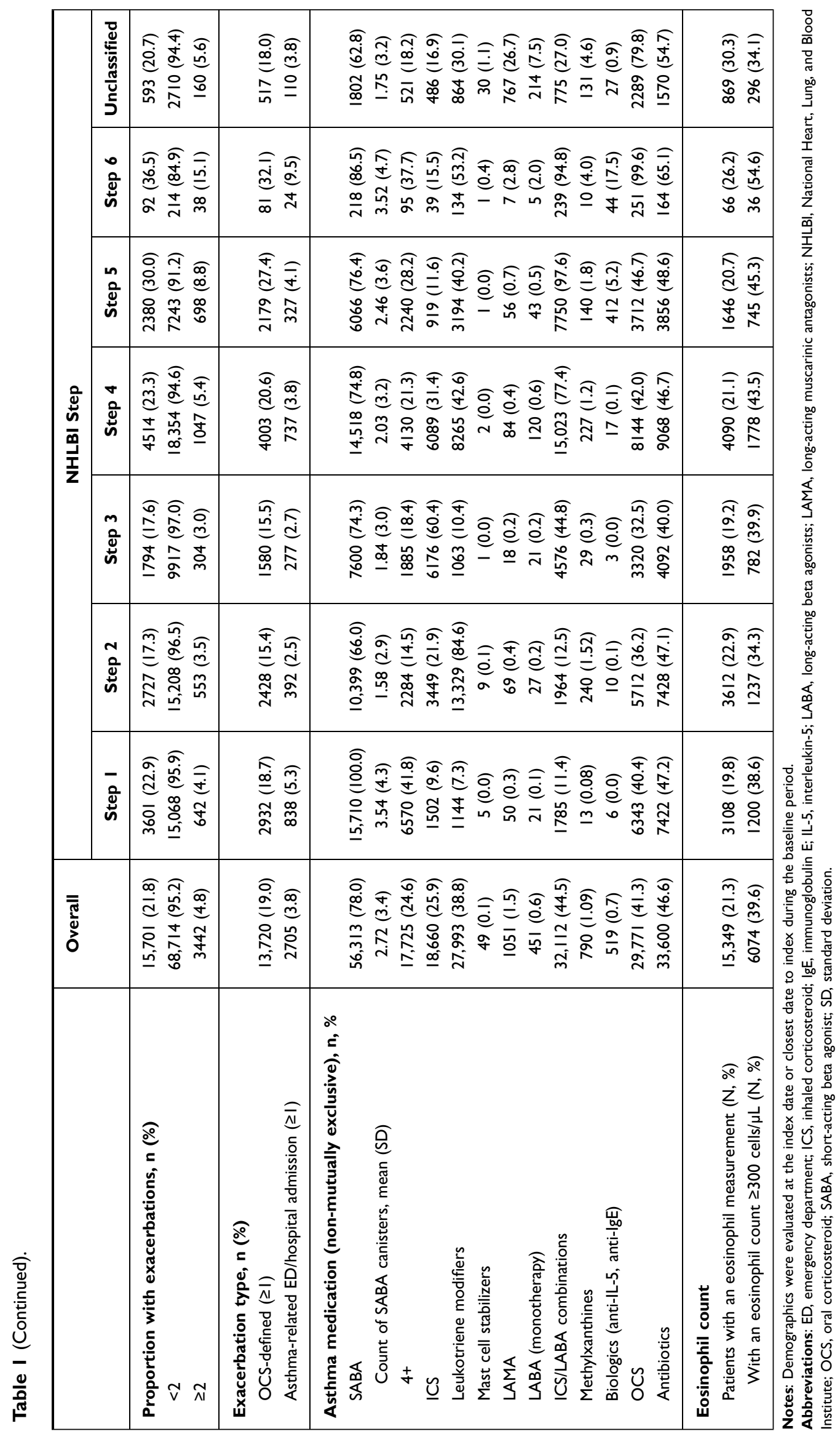




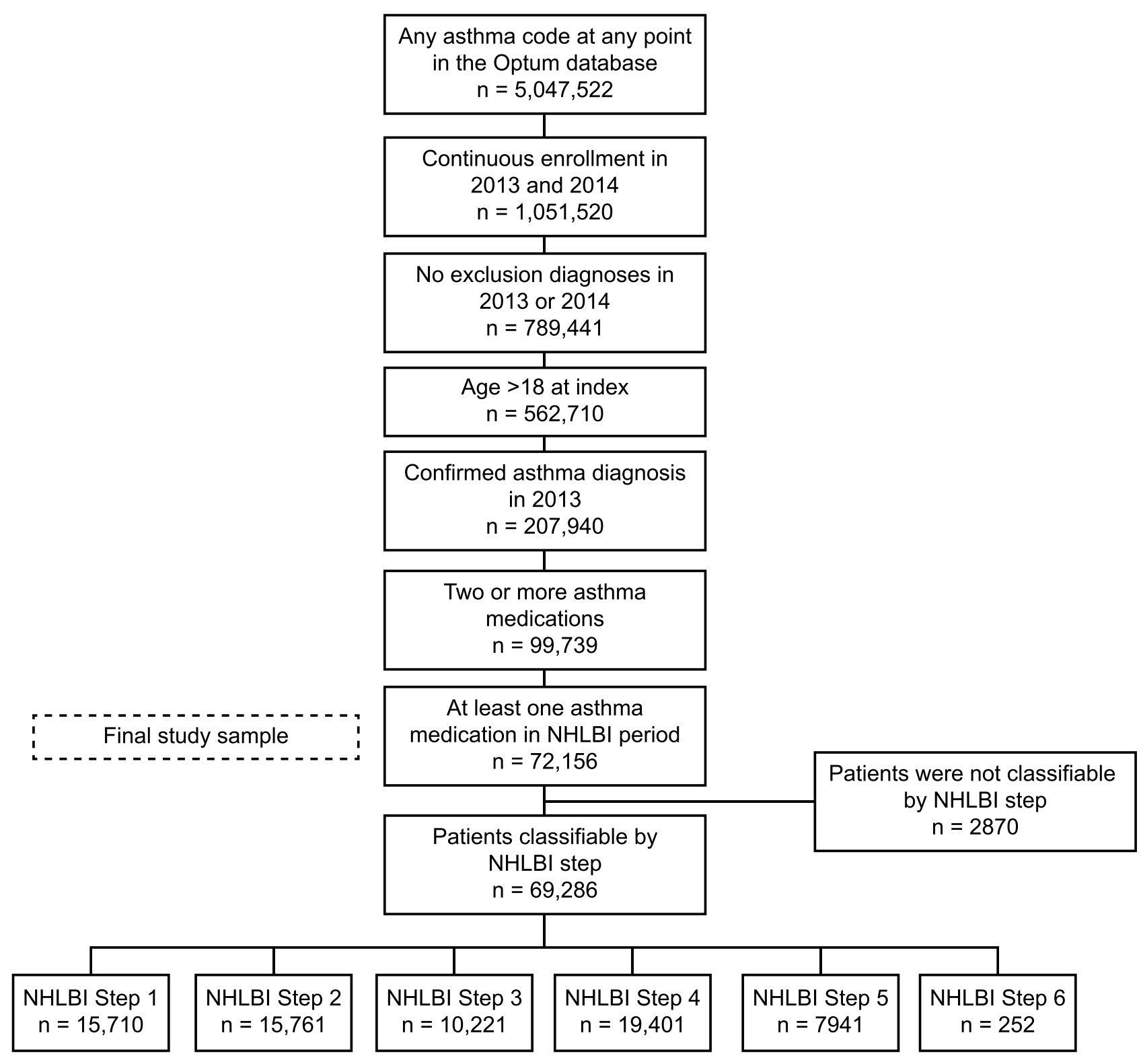

Figure 2 Patient flow diagram - describes patient attrition throughout the study and step classification. Abbreviaton: NHLBI, National Heart, Lung, and Blood Institute.

related healthcare encounters $(62.9 \%$ for outpatient visits, $8.0 \%$ for ED visits, and $1.7 \%$ for hospitalizations) when compared with the overall study population. Also, among patients with an exacerbation, there were higher proportions of patients with allergic rhinitis during the baseline period compared with the overall population $(46.4 \%$ vs $40.1 \%)$.

\section{NHLBI Asthma Treatment Steps}

A total of 69,286 patients met the criteria to be classified within an NHLBI step; $2870(4.0 \%)$ patients were not classifiable (Table 1). Approximately $44 \%$ of patients were classified as NHLBI Steps $1-2 ; 41 \%$ as NHLBI Steps 3-4; and $11 \%$ as Steps 5-6. There was an increase from Steps 2-6 in the percent of patients with at least one prior exacerbation during the baseline period. Of the patients with an eosinophil value available (19.2-26.2\%, depending on step), the proportion of patients with an eosinophil count $\geq 300$ cells $/ \mu \mathrm{L}$ generally increased with NHLBI step. Among patients in the exacerbating cohort $(\geq 1$ exacerbation in the follow-up period), the classification of patients by NHLBI step had relatively more patients in Steps 4-6 and slightly fewer patients in Steps 1-3 compared with 


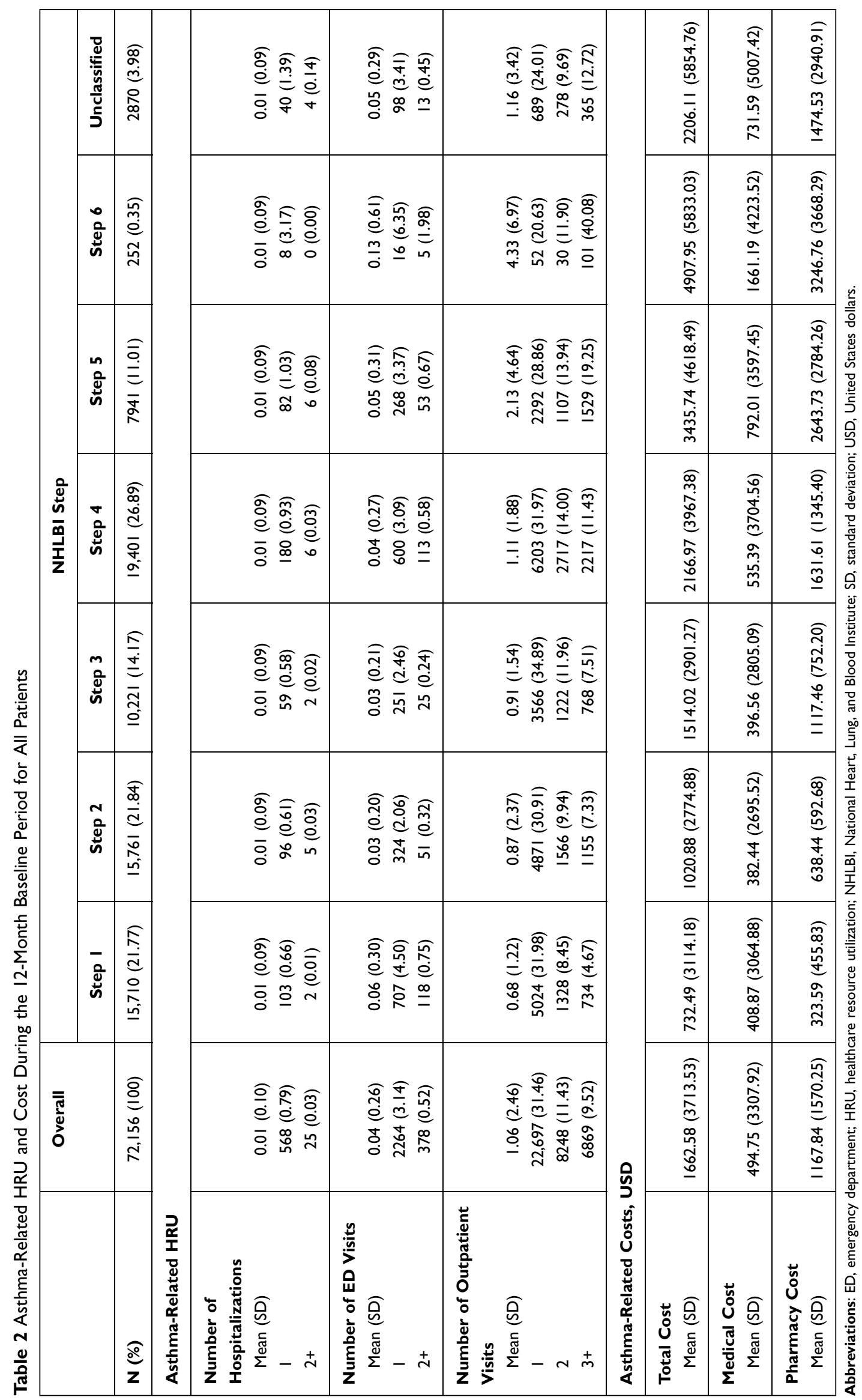


the overall population: Step $1(n=2163,20.4 \%)$; Step 2 $(n=1931,18.2 \%)$; Step $3(n=1280,12.1 \%)$; Step 4 $(n=3121,29.5 \%)$; Step $5(n=1641,15.5 \%)$; and Step 6 $(n=66,0.6 \%)$.

\section{Asthma Exacerbations in the Follow-Up Period}

A total of $10,590(14.8 \%)$ patients experienced at least one exacerbation during the follow-up period and had a mean exacerbation rate of 1.36 (SD 0.79) exacerbations per year. At least $13 \%$ of the total study population experienced an OCS-defined exacerbation while $2.2 \%$ had at least one asthma-related ED visit/hospital admission-defined exacerbation (Supplementary Table 2). Exacerbations were observed among patients classified at each of the NHLBI steps and were more frequent with increasing step (Figure 3). The baseline exacerbation rate was higher among patients who experienced an exacerbation in the follow-up period compared with the overall population (0.29 [SD 0.64] vs 0.67 [SD 1.01]).

Similar trends were observed for OCS-defined and asthma-related ED/hospital admission exacerbations; however, Step 1 patients had a higher proportion of ED/hospital admission exacerbations (3.1\%) compared with Steps $2-5(1.6 \%$ to $2.4 \%)$ (Supplementary Table 2). Of note, $6.8 \%$ of Step 5 and $9.9 \%$ of Step 6 patients experienced at least two exacerbations during the follow-up period.

With respect to seasonality, the majority of exacerbations occurred in the winter and spring (30.8\% and $26.1 \%$,

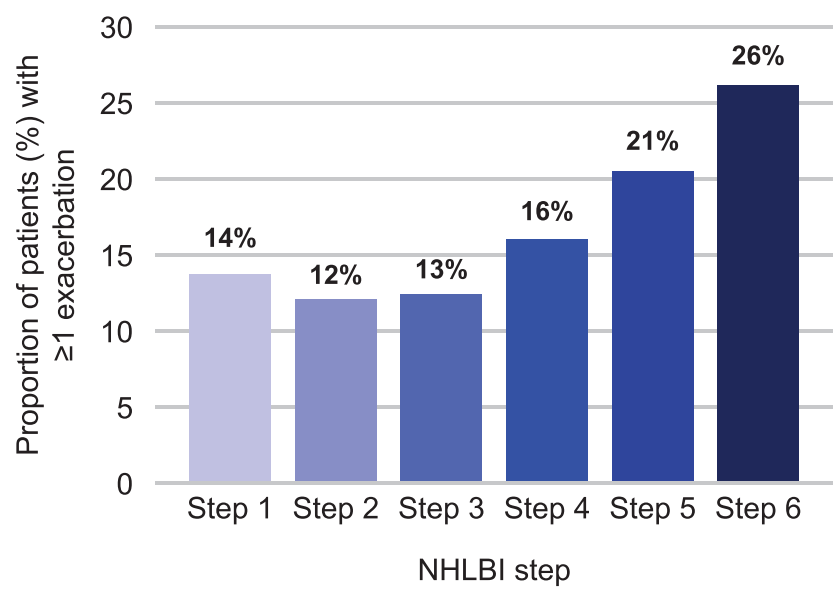

Figure 3 Exacerbations during the follow-up period (2014) by NHLBI step $(n=10,590)$ - bar chart shows the proportion of patients who experienced an exacerbation in the follow-up period, classified by NHLBI step.

Abbreviations: NHLBI, National Heart, Lung, and Blood Institute. respectively) followed by the fall and to a lesser extent in the summer $(24.0 \%$ and $19.1 \%$, respectively $)$ (Supplementary Table 2).

\section{Healthcare Resource Utilization in the Follow-Up Period}

In the overall population, the mean annual number of allcause hospitalizations (all steps: 0.08, SD 0.36) and outpatient visits (all steps: 12.29, SD 14.15) in the follow-up period were similar across Steps $1-5$, and highest among patients at Step 6 (hospitalizations [0.13, SD 0.44], outpatient visits [19.33, SD 16.46]). Asthma-related HRU increased by NHLBI step and was highest for Step 6 for mean asthmarelated hospitalizations ( 0.03 , SD 0.20$)$, ED visits $(0.06$, SD 0.27 ), and outpatient visits (4.38, SD 7.99) (Table 3). A similar pattern was seen for the percent of patients with at least one asthma-related hospitalization, ED visit, and outpatient visit, as shown in Figure 4. In addition, the mean number and percentage of patients with at least one asthmarelated ED visit were higher among Step 1 patients (mean 0.04, SD 0.28; 3.1\%) than those at Steps 2-5.

In the exacerbating population, mean rates for all-cause hospitalizations, ED visits, and outpatient visits were 0.11 (SD 0.41), 0.70 (SD 1.53), and 15.69 (SD 15.54), respectively, which were higher than those for the overall asthma population at all steps except for all-cause hospitalizations and ED visits at Step 6. Among patients with at least one exacerbation in the follow-up, total asthma-related HRU was highest for Step 6 (8.00, SD 9.83) and next highest for Step 5 (3.51, SD 6.10). Exacerbators in Step 6 had the highest mean number of hospitalizations (0.05, SD 0.21). Unexpectedly, Step 1 exacerbators had the highest mean number of ED visits $(0.30$, SD 0.70$)$ during the follow-up period, possibly suggesting a lack of control or under-treatment.

\section{Healthcare Costs in the Follow-Up Period}

In the overall treated asthma population, mean annual allcause total costs were $\$ 11,673.09$ (SD $\$ 23,951.48$ ) and mean asthma-related total costs were $\$ 1367.80$ (SD \$3910.96). Mean asthma-related total costs increased with each NHLBI step (Figure 5).

Asthma-related pharmacy and medical costs for Step 6 patients (\$3251.04 and \$1627.29, respectively) were at least three times higher than the average costs in the total population ( $\$ 1018.54$ and $\$ 349.25$, respectively) (Table 3). 
In the exacerbating population, mean all-cause total costs were $\$ 14,834.92$ (SD $\$ 25,569.54$ ) and mean asthma-related total costs were $\$ 2560.22$ (SD \$4534.78). Mean costs among exacerbators also increased by NHLBI step and were markedly higher in all asthma-related cost categories compared with the overall asthma population. Mean asthma-related total, medical, and pharmacy costs among patients with at least one exacerbation in the follow-up were highest for Step 6 (total $\$ 6372.10$; medical \$2897.22; pharmacy \$3474.88) and next highest for Step 5 (total \$4176.88, medical \$1330.09, pharmacy \$2846.79).

\section{Medication Adherence and Treatment Patterns}

In the overall population during the baseline period, the mean number of SABA canisters dispensed was 2.72 (SD 3.37), $78.0 \%$ of patients had at least one SABA dispensing, $41.3 \%$ had at least one OCS dispensing, and their use generally increased with NHLBI step (Table 1). Use of SABA and OCS was higher among the exacerbating cohort (both at baseline and during the follow-up period; data not shown). With regards to rescue medication use in the overall population during the follow-up period, the mean number of SABA canisters dispensed was 2.28 (SD 3.51) and was lower than in the exacerbating subgroup (3.63, SD 4.37) (Supplementary Tables 3 and 4).

Adherence in the follow-up period, as measured by mean PDC (0.59 vs 0.56 , respectively), was slightly higher in the overall population compared with the exacerbating population; although the reverse was observed with the proportion of adherent patients, as measured by PDC $\geq 80 \% \quad(28.7 \% \quad$ vs $32.3 \%)$ (Supplementary Tables 3 and 4). For both measures, adherence to maintenance therapy tended to increase with increasing NHLBI step. The mean AMR was 0.65 in the overall population versus 0.60 in the exacerbation population (Supplementary Tables 3 and 4). Maintenance therapy discontinuation rates tended to be high for both the overall and exacerbating populations, and they also tended to decrease with increasing NHLBI step.

In the overall population, a quarter of all Step 1 patients $(25.9 \%)$ escalated to Steps 2-4 during the follow-up period while $9.9 \%$ of patients in Steps 2-4 escalated to Steps 5-6 (Supplementary Table 3). Among those with exacerbations, more Step 1 patients (48.3\%) escalated to Steps 2-4, and almost twice as many Step 2-4 patients escalated to Steps 5-6 (18.0\%), compared with the overall population (Supplementary Table 4).

\section{Discussion}

This study examined a large asthma cohort $(n=72,156)$ in the US. To our knowledge, it is the first study to quantify the burden of exacerbations occurring among the treated asthma population using the NHLBI step categorization as a proxy measure for disease severity. Among the overall cohort of treated asthma patients, a total of 10,590 (14.7\%) experienced one or more exacerbations in the follow-up period. This population had clinical characteristics that were notably different from the overall study population: a higher proportion of patients had experienced comorbid allergic rhinitis, had blood eosinophil counts $\geq 300$ cells/ $\mu \mathrm{L}$, and had asthma-related healthcare encounters during the baseline period, compared with the overall population.

During the 12-month follow-up period, asthma-related exacerbations, HRU, and costs increased with increasing NHLBI step (severity). In addition, the proportion of patients who were adherent to their asthma maintenance medication ( $\mathrm{PDC} \geq 80 \%$ ) was low for both the overall and exacerbating cohorts $(28.7 \%$ and $32.0 \%$, respectively); however, higher adherence was observed with increasing NHLBI steps. Treatment discontinuation rates were also high for both the overall and exacerbating cohorts but were progressively lower with increasing NHLBI step. The use of rescue medications was more frequent in the exacerbating cohort compared with the overall population and increased with NHLBI step.

Exacerbations were observed among patients at all NHLBI steps and were more frequent with increasing step/asthma severity. Patients who experienced an exacerbation in the follow-up period also had a higher rate of baseline exacerbations compared with the overall population. This aligns with previous findings where asthma patients with an exacerbation in the prior year (vs those without) had 7.80-times higher odds of experiencing an exacerbation in the subsequent year. ${ }^{25}$ Seasonality trends indicated a higher frequency of exacerbations in the winter/spring, followed by the fall, and lowest in the summer - despite exacerbations remaining prevalent during this season. The proportion of patients escalating to a higher step in the exacerbating population was as expected compared with the overall population: nearly double the percentage of patients escalated from Step 1 to Steps $2-4(48.3 \%$ vs $25.9 \%)$ and from Steps $2-4$ to Steps 5-6 (18.0\% vs 9.9\%). 


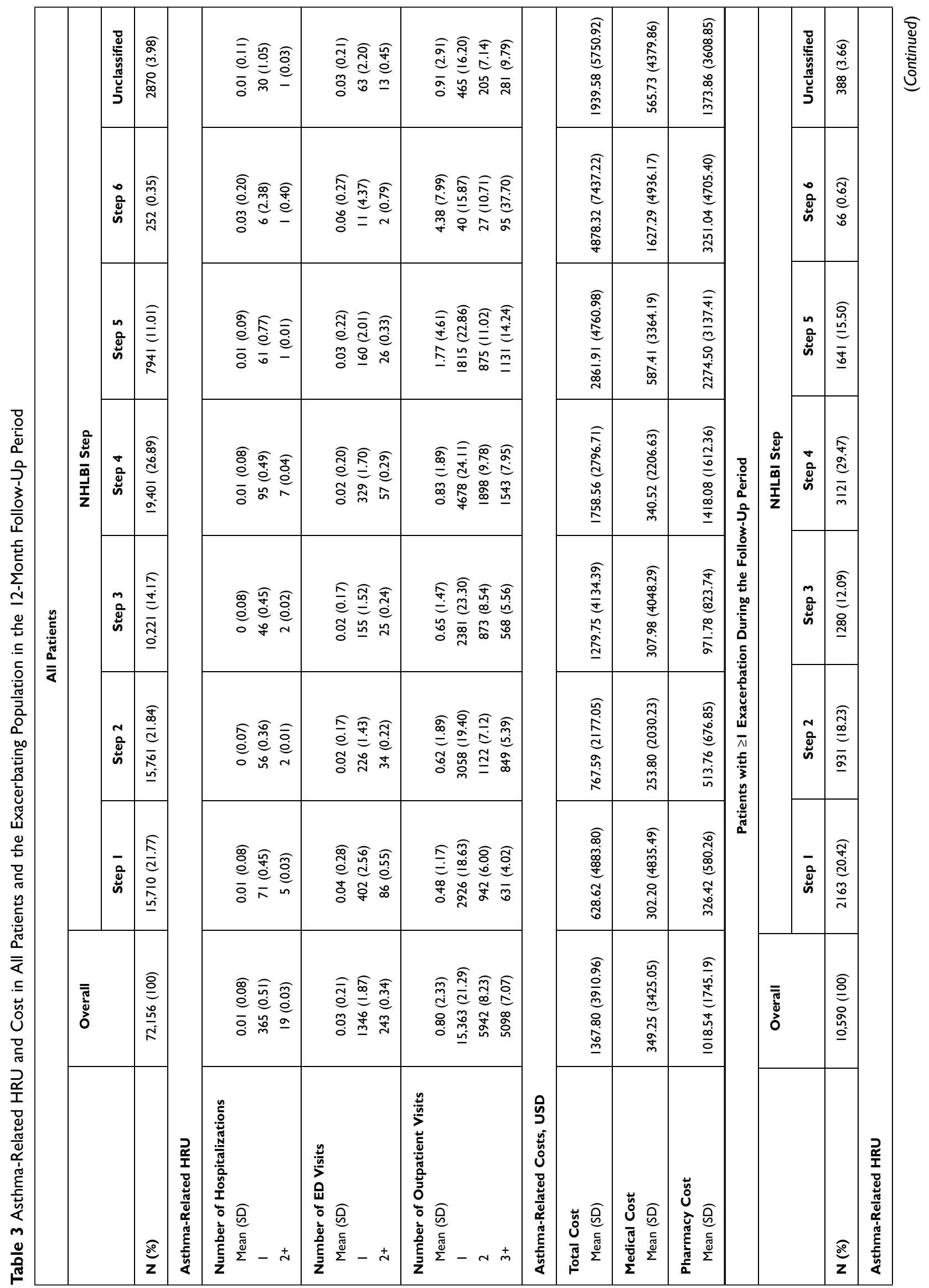




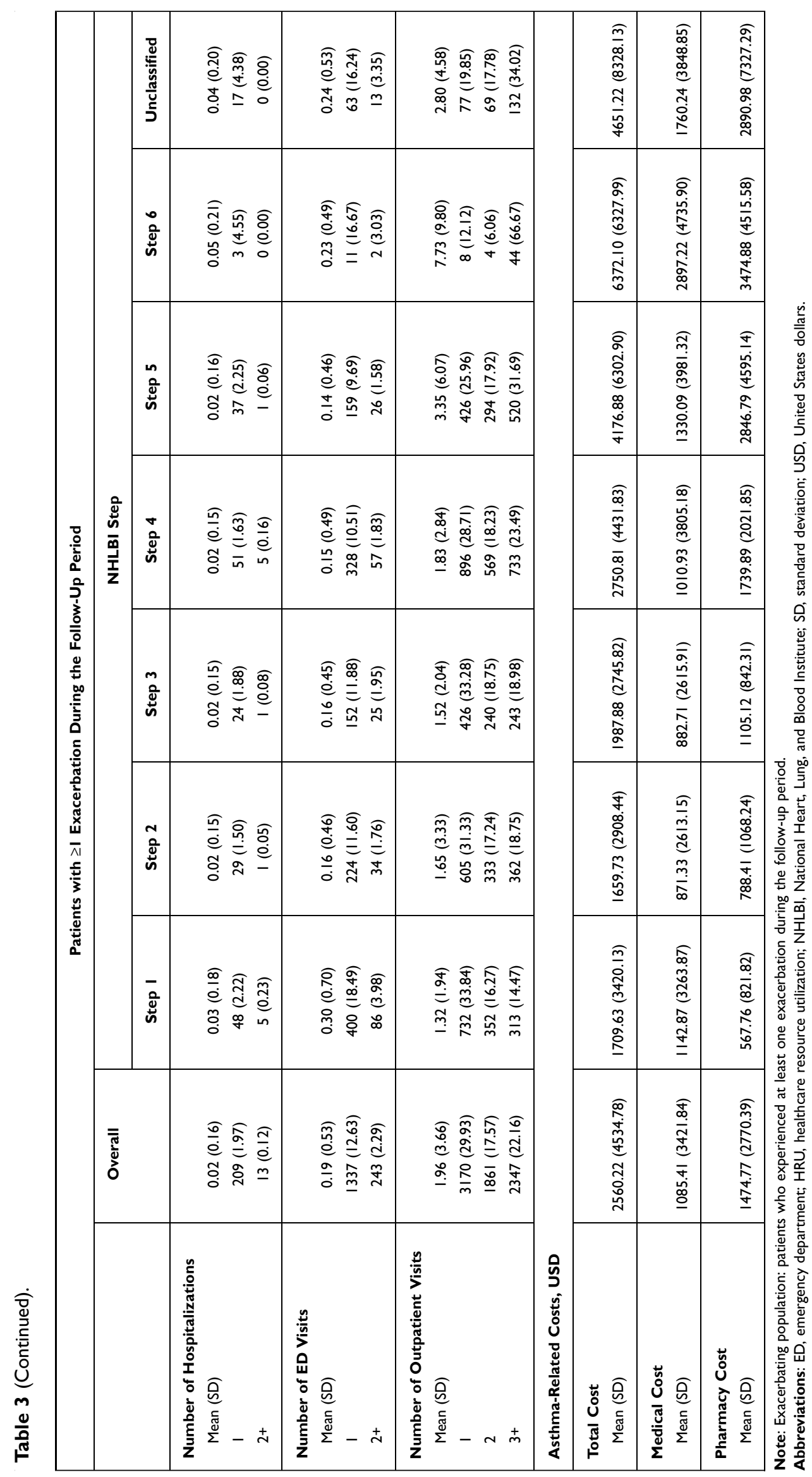


A

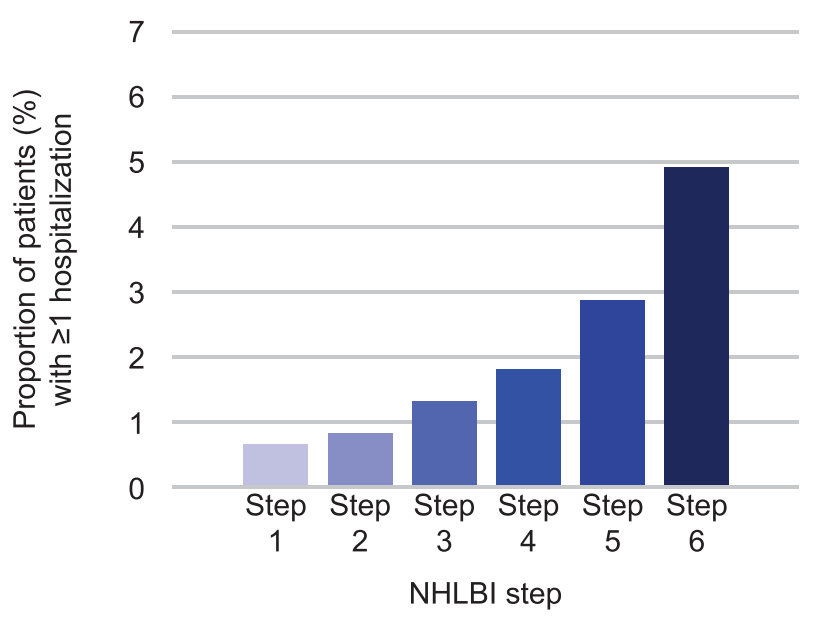

B

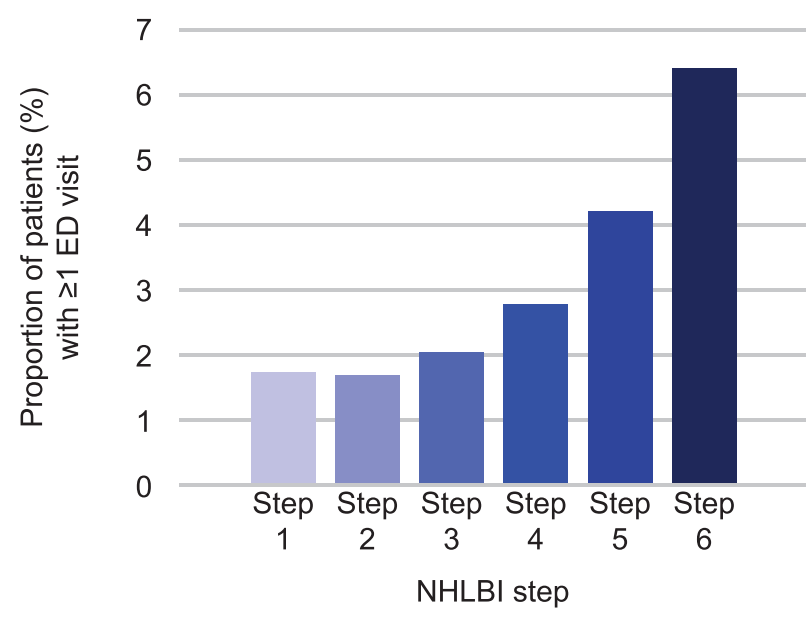

C

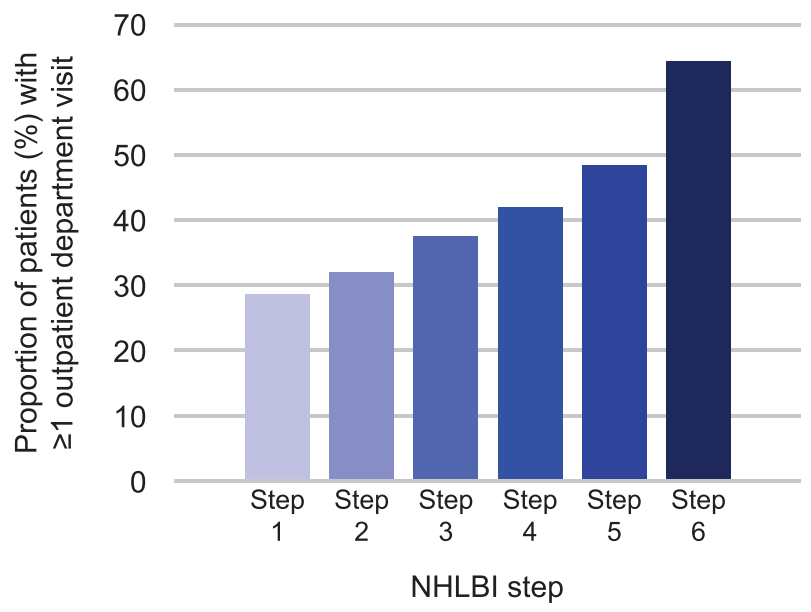

Figure 4 Asthma-related HRU during the follow-up period (2014) by NHLBI step for the overall population $(\mathrm{N}=72,156)$ - bar charts illustrate the proportion of all patients who required hospitalization (A), ED visit (B), or outpatient department visit (C) during the follow-up period, classified by NHLBI step.

Abbreviations: ED, emergency department; HRU, healthcare resource utilization; NHLBI, National Heart, Lung, and Blood Institute.

These study results further concur with an earlier retrospective cohort study, which reported on asthma exacerbations and healthcare use by asthma severity, as defined by the GINA step classification. ${ }^{22}$ Suruki and colleagues reported that the percentage of treated asthma patients with $\geq 1$ exacerbation in the follow-up period was $12.5 \%$ in their US database (vs $14.7 \%$ in the current study) and mean exacerbation rate was 0.16 per year within this population (vs 0.20 per year in our study). Similar trends were also seen, with exacerbations occurring more frequently in patients with increasing GINA step/disease severity, and in those with clinical traits of allergic rhinitis (atopy) and elevated eosinophil levels. ${ }^{22}$ Patients in GINA Step 5 (eg, the most severe group, akin to NHLBI Steps
5-6) had the highest rate of exacerbations and mean total HRU, and total all-cause and asthma-related costs per exacerbation in the 30 days after an exacerbation. These patterns were consistent with those observed in our study, including the increases in these parameters according to severity. Our study shared multiple characteristics with the study by Suruki et al; both studies analyzed a 1-year baseline and 1-year follow-up period, utilized the Optum dataset, utilized similar exacerbation definitions, and considered treatments during a 90-day window to categorize treatment step (GINA/NHLBI). The primary differences between the studies were those between GINA and NHLBI classifications, as well as the study time periods. Regarding study design, the current study utilized calendar 
A

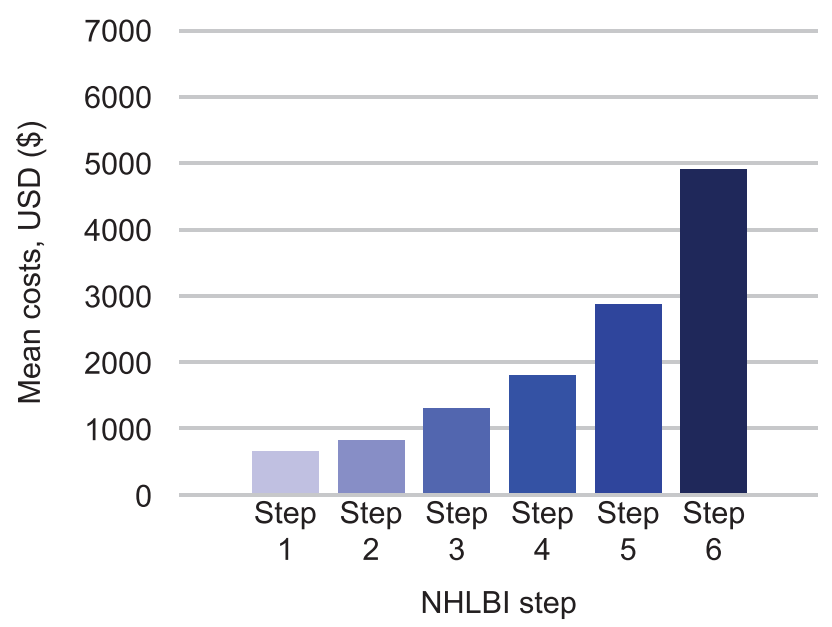

B

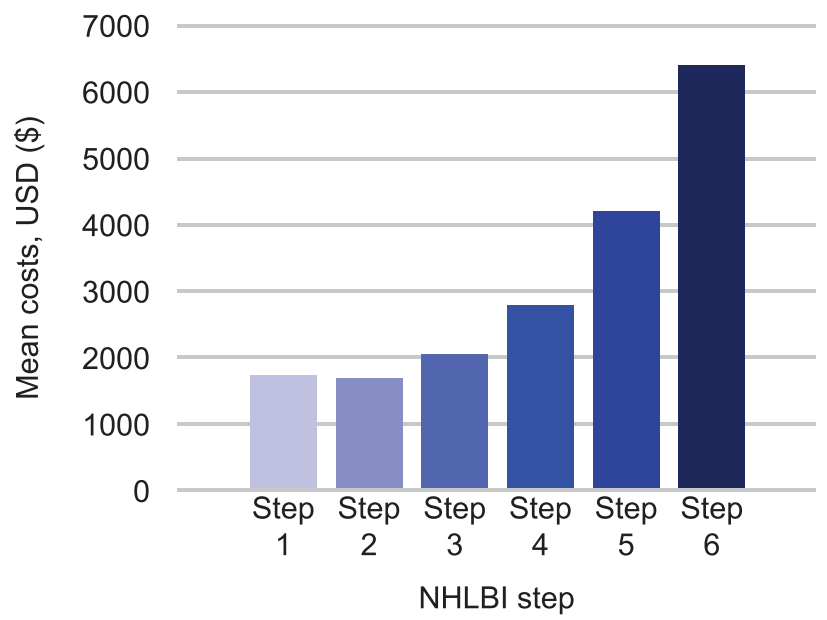

Figure 5 Asthma-related total costs during the follow-up period (2014) by NHLBI step - bar charts illustrate the cost associated with patients in each NHLBI step for both the overall population (A) and for those who experienced $\geq$ I exacerbation during follow-up (B). Costs were adjusted to 2016 USD (\$).

Abbreviations: NHLBI, National Heart, Lung, and Blood Institute; USD, United States dollars.

years to ensure that any seasonal trends in exacerbations were captured; whereas, Suruki et al identified an index date based on an asthma diagnosis during the study period, therefore the baseline and follow-up periods for individual patients may have varied in the seasons/months that were encompassed.

NHLBI Step 1 patients (with intermittent asthma), when compared with NHLBI Step 2 and 3 patients (mild-moderate asthma), had a slightly higher mean number of exacerbations, ED visits, and higher SABA use during the baseline period. This may indicate that those in NHLBI Step 1 had poor control of their asthma, and thus could potentially benefit from earlier use of inhaled maintenance therapy to better control their asthma. This suggestion is supported by the finding that over a quarter (25.9\%) of NHLBI Step 1 patients stepped up to NHLBI Steps 2-4 therapy in the follow-up period. Notably, since 2019, GINA no longer recommends SABA only use but rather ICS/LABA as needed as reliever treatment for patients with intermittent asthma; this is in discordance with the latest NHLBI 2020 guidelines which continue to recommend SABA use as needed for intermittent asthma in Step 1. Another explanation for this finding could be that the study inclusion criteria required a prescription for asthma medication within 90 days of the index date, which, in the context of intermittent asthma, would be a rescue inhaler. This could have potentially excluded those with well-controlled asthma and increased the likelihood of identifying uncontrolled or worsening intermittent asthma. The highest exacerbation rate was still observed in patients with asthma categorized as NHLBI Steps 5-6.

Direct total and asthma-related costs in the 12-month follow-up period also increased with NHLBI step and were highest for more severe patients at NHLBI Steps 5-6; this was in agreement with previous studies. ${ }^{15,26}$ Some studies have detailed further indirect costs and highlighted the unmet need for severe asthma patients, as evidenced by patient questionnaires, health-related quality of life, work productivity, and activity impairment. ${ }^{27-29}$

Considered together, our study findings highlight a large disease burden and continued unmet need in the treated US asthma population. Additional research and improved multifactorial assessment strategies may help to address the underlying drivers of disease and improve the management of asthma. For example, severe refractory eosinophilic airway inflammation in asthma is a clinically valid endotype associated with frequent exacerbations. ${ }^{30} \mathrm{It}$ has been described as a treatable trait since it is identifiable, measurable, and treatable, allowing for targeted therapy to improve outcomes for individual patients. ${ }^{31}$ In this study, characterization of patients according to clinical traits was not widespread; we note that blood eosinophil levels were not measured in the majority of patients, with only $21.3 \%$ of the overall population having an eosinophil measurement. Through incorporating exacerbation risk and blood eosinophil count into a multidimensional assessment, there is an opportunity to identify those patients for 
who appropriately targeted treatment could help drive better disease outcomes. $^{31-33}$

Limitations of this study include those inherent to the use of retrospective claims database methodology, such as the lack of clinical measures of disease severity and symptom control, for which the characterization by NHLBI steps served as a proxy. As the Optum database contains a large sample of individuals in the US covered by private insurance plans, the results of this study may not be generalizable to US patients who receive healthcare through government organizations (eg, Medicare, Medicaid), other commercially insured populations, or to those without health insurance. Our findings also reflect asthma management practices among US physicians and may not, therefore, be generalizable to other countries; additionally, the study data represent only patients who have sought care or treatment for asthma. Furthermore, the NHLBI steps are based on guidelines for asthma management and are intended for dynamic application to maximize control of disease. The current study attempted to discern the level of asthma control by retrospectively determining the extent of asthma treatment for each patient during the 90-day period prior to the index date. As a result, there is potential for misclassification of asthma control level (NHLBI steps) because the claims database may not accurately reflect the clinical presentation and/or control of the disease. Since exacerbations in this study are more common in winter, it is reasonable to suspect a significant amount of people would have an exacerbation in the 90 days prior to index, which in turn may have affected treatment step determination. A further limitation linked to the use of NHLBI steps as a proxy for severity is that those who were receiving medications not consistent with any of the steps remained unclassified; however, over $96 \%$ of patients were classified. As the data examined in this study were derived from 2013-2014, our findings may not fully reflect the current situation in the treated US asthma population. The healthcare costs may also not reflect the current landscape as, being adjusted to 2016 US dollars, they have likely further inflated over the past few years.

The strengths of this study include its use of a large integrated US health insurance claims database (ie, capturing the whole patient encounter record), thus allowing observation of, and real-world insights on, a large number of patients with asthma, diverse medical histories, and varying disease severity. Administrative claims data are a rich data source for examining patterns of care and health outcomes in a real-world setting. As the health plans included in the database span a wide geographic distribution across the US, it provides the capability for generalization of the study results to managed care populations on a national level.

\section{Conclusions}

Exacerbations were observed among treated asthma patients classified at each of the NHLBI steps (used as a proxy for disease severity) and were more frequent with increasing step categories. Asthma-related HRU and associated costs also increased with increasing NHLBI step categories and were higher among those who experienced one or more exacerbations. These trends in exacerbations and asthma-related HRU highlight the continued unmet need within the treated US asthma population.

\section{Abbreviations}

AMR, asthma medication ratio; ED, emergency department; GINA, Global Initiative for Asthma; HRU, healthcare resource utilization; ICD-9, International Classification of Diseases, 9th revision; ICS, inhaled corticosteroid; NHLBI, National Heart, Lung, and Blood Institute; OCS, oral corticosteroid; PDC, proportion of days covered; SABA, short-acting beta agonist; SD, standard deviation; US, United States.

\section{Data Sharing Statement}

The data contained in our database contains proprietary elements owned by Optum and, therefore, cannot be broadly disclosed or made publicly available at this time. The disclosure of these data to third-party clients assumes certain data security and privacy protocols are in place, and that the third-party client has executed our standard license agreement which includes restrictive covenants governing the use of the data.

\section{Ethics Approval and Consent to Participate}

This study used de-identified claims data, and as such, this study did not require Institutional Review Board approval. This data was used with permission from Optum. GSK complies with laws and regulations to ensure data privacy and appropriate use of de-identified datasets for research, including the Health Insurance Portability and Accountability Act of 1996 (HIPAA), in order to protect 
sensitive patient health information from being disclosed without the patient's consent or knowledge.

\section{Acknowledgments}

Editorial support (in the form of editorial suggestions to draft versions of this paper, assembling tables and figures, collating author comments, copyediting, referencing, and graphic services) was provided by Kyle Kennedy, MBChB, of Ashfield MedComms (Macclesfield, UK), an Ashfield Health company, and was funded by GlaxoSmithKline plc. Trademarks are owned by or licensed to their respective owners (GlaxoSmithKline plc. or Optum). The abstract of this paper was presented at the American Thoracic Society 2019 International Conference as a poster presentation with interim findings. The poster's abstract was published in "Conference Abstracts" in American Journal of Respiratory and Critical Care Medicine: https://doi.org/10.1164/ajrccmconference.2019.199.1_MeetingAbstracts.A4060.

\section{Author Contributions}

All authors made substantial contributions to conception and design, acquisition of data, or analysis and interpretation of data; took part in drafting the article or revising it critically for important intellectual content; agreed to submit to the current journal; gave final approval of the version to be published; and agree to be accountable for all aspects of the work.

\section{Funding}

This work was funded by GlaxoSmithKline plc. (study HO-16-16511).

\section{Disclosure}

All authors are shareholders in GlaxoSmithKline plc.; CMA and JF-B are employees of GlaxoSmithKline plc., and $\mathrm{DH}, \mathrm{BW}$, and RL were employed by GlaxoSmithKline plc. at the time this study was conducted. The authors report no other conflicts of interest in this work.

\section{References}

1. Akinbami LJ, Moorman JE, Bailey C, et al. Trends in asthma prevalence, health care use, and mortality in the United States, 2001-2010. NCHS Data Brief. 2012;94:1-8.

2. Enilari O, Sinha S. The global impact of asthma in adult populations. Ann Glob Health. 2019;85(1):2. doi:10.5334/aogh.2412

3. Colice GL. Categorizing asthma severity: an overview of national guidelines. Clin Med Res. 2004;2(3):155-163. doi:10.3121/ cmr.2.3.155

4. Dougherty RH, Fahy JV. Acute exacerbations of asthma: epidemiology, biology and the exacerbation-prone phenotype. Clin Exp Allergy. 2009;39(2):193-202. doi:10.1111/j.1365-2222.2008.03157.x
5. Accordini S, Bugiani M, Arossa W, et al. Poor control increases the economic cost of asthma. A multicentre population-based study. Int Arch Allergy Immunol. 2006;141(2):189-198. doi:10.1159/ 000094898

6. Accordini S, Corsico AG, Braggion M, et al. The cost of persistent asthma in Europe: an international population-based study in adults. Int Arch Allergy Immunol. 2013;160(1):93-101. doi:10.1159/000338998

7. Rodrigo GJ, Rodrigo C, Hall JB. Acute asthma in adults: a review. Chest. 2004;125(3):1081-1102. doi:10.1378/chest.125.3.1081

8. Serra-Batlles J, Plaza V, Morejón E, Comella A, Brugués J. Costs of asthma according to the degree of severity. Eur Respir J. 1998;12 (6):1322-1326. doi:10.1183/09031936.98.12061322

9. Global Initiative for Asthma (GINA). Global strategy for asthma management and prevention; 2020. Available from: https:// ginasthma.org/wp-content/uploads/2020/04/GINA-2020-full-report_final-_wms.pdf. Accessed June, 2020.

10. Globe G, Martin M, Schatz M, et al. Symptoms and markers of symptom severity in asthma-content validity of the asthma symptom diary. Health Qual Life Outcomes. 2015;13:21. doi:10.1186/s12955015-0217-5

11. Zervas E, Samitas K, Papaioannou AI, Bakakos P, Loukides S, Gaga M. An algorithmic approach for the treatment of severe uncontrolled asthma. ERJ Open Res. 2018;4(1):00125-2017. doi:10.1183/ 23120541.00125-2017

12. Global Initiative for Asthma (GINA). Difficult-to-treat \& severe asthma in adolescent and adult patients: diagnosis and management; 2018. Available from: https://ginasthma.org/wp-content/uploads/ 2019/04/GINA-Severe-asthma-Pocket-Guide-v2.0-wms-1.pdf. Accessed June, 2020.

13. Griswold SK, Nordstrom CR, Clark S, Gaeta TJ, Price ML, Camargo CA Jr. Asthma exacerbations in North American adults: who are the "frequent fliers" in the emergency department? Chest. 2005;127(5):1579-1586. doi:10.1378/chest.127.5.1579

14. Guilbert TW, Garris C, Jhingran P, et al. Asthma that is not well-controlled is associated with increased healthcare utilization and decreased quality of life. J Asthma. 2011;48(2):126-132. doi:10.3109/02770903.2010.535879

15. Ivanova JI, Bergman R, Birnbaum HG, Colice GL, Silverman RA, McLaurin K. Effect of asthma exacerbations on health care costs among asthmatic patients with moderate and severe persistent asthma. $J$ Allergy Clin Immunol. 2012;129(5):1229-1235. doi:10.1016/j.jaci.2012.01.039

16. Bateman ED, O'Byrne PM, Busse WW, et al. Once-daily fluticasone furoate $(\mathrm{FF}) /$ vilanterol reduces risk of severe exacerbations in asthma versus FF alone. Thorax. 2014;69(4):312-319. doi:10.1136/thoraxjnl2013-203600

17. Jackson DJ, Sykes A, Mallia P, Johnston SL. Asthma exacerbations: origin, effect, and prevention. J Allergy Clin Immunol. 2011;128 (6):1165-1174. doi:10.1016/j.jaci.2011.10.024

18. O’Byrne PM, Barnes PJ, Rodriguez-Roisin R, et al. Low dose inhaled budesonide and formoterol in mild persistent asthma: the OPTIMA randomized trial. Am J Respir Crit Care Med. 2001;164(8 Pt 1):1392-1397. doi:10.1164/ajrccm.164.8.2104102

19. Pauwels RA, Löfdahl CG, Postma DS, et al. Effect of inhaled formoterol and budesonide on exacerbations of asthma. Formoterol and Corticosteroids Establishing Therapy (FACET) International Study Group. N Engl J Med. 1997;337(20):1405-1411. doi:10.1056/ NEJM199711133372001

20. National Heart, Lung, and Blood Institute (NHLBI). National Asthma Education and Prevention Program (NAEPP); 2020. Available from: https://www.nhlbi.nih.gov/science/national-asthma-education-and-pre vention-program-naepp. Accessed August, 2020.

21. National Heart, Lung, and Blood Institute (NHLBI). Expert panel report 3: guidelines for the diagnosis and management of asthma; 2007. Available from: https://www.nhlbi.nih.gov/health-topics/guide lines-for-diagnosis-management-of-asthma. Accessed June, 2020. 
22. Suruki RY, Daugherty JB, Boudiaf N, Albers FC. The frequency of asthma exacerbations and healthcare utilization in patients with asthma from the UK and USA. BMC Pulm Med. 2017;17(1):74. doi:10.1186/s12890-017-0409-3

23. Schatz M, Nakahiro R, Crawford W, Mendoza G, Mosen D, Stibolt TB. Asthma quality-of-care markers using administrative data. Chest. 2005;128(4):1968-1973. doi:10.1378/chest.128.4.1968

24. Raebel MA, Schmittdiel J, Karter AJ, Konieczny JL, Steiner JF. Standardizing terminology and definitions of medication adherence and persistence in research employing electronic databases. Med Care. 2013;51(8 Suppl 3):S11-21. doi:10.1097/MLR.0b013 e31829b1d2a

25. Schatz M, Meckley LM, Kim M, Stockwell BT, Castro M. Asthma exacerbation rates in adults are unchanged over a 5-year period despite high-intensity therapy. $J$ Allergy Clin Immunol Pract. 2014;2(5):570-574.e1. doi:10.1016/j.jaip.2014.05.002

26. Zeiger RS, Schatz M, Dalal AA, et al. Utilization and costs of severe uncontrolled asthma in a managed-care setting. $J$ Allergy Clin Immunol Pract. 2016;4(1):120-129.e3. doi:10.1016/j. jaip.2015.08.003

27. Davis J, Trudo F, Siddall J, Small M. Burden of asthma among patients adherent to ICS/LABA: a real-world study. J Asthma. 2019;56(3):332-340. doi:10.1080/02770903.2018.1455858
28. Lee LK, Obi E, Paknis B, Kavati A, Chipps B. Asthma control and disease burden in patients with asthma and allergic comorbidities. J Asthma. 2018;55(2):208-219. doi:10.1080/02770903.2017.1316394

29. Stone B, Davis JR, Trudo F, et al. Characterizing patients with asthma who received Global Initiative for Asthma steps 4-5 therapy and managed in a specialty care setting. Allergy Asthma Proc. 2018;39(1):27-35. doi:10.2500/aap.2018.39.4094

30. Gibson PG, McDonald VM. Management of severe asthma: targeting the airways, comorbidities and risk factors. Intern Med J. 2017;47 (6):623-631. doi:10.1111/imj.13441

31. McDonald VM, Fingleton J, Agusti A, et al. Treatable traits: a new paradigm for 21 st century management of chronic airway diseases: Treatable Traits Down Under International Workshop report. Eur Respir J. 2019;53(5):1802058. doi:10.1183/13993003.02058-2018

32. Agusti A, Bel E, Thomas M, et al. Treatable traits: toward precision medicine of chronic airway diseases. Eur Respir J. 2016;47 (2):410-419. doi:10.1183/13993003.01359-2015

33. Pavord ID, Agusti A. Blood eosinophil count: a biomarker of an important treatable trait in patients with airway disease. Eur Respir J. 2016;47(5):1299-1303. doi:10.1183/13993003.00055-2016

\section{Publish your work in this journal}

The Journal of Asthma and Allergy is an international, peer-reviewed open-access journal publishing original research, reports, editorials and commentaries on the following topics: Asthma; Pulmonary physiology; Asthma related clinical health; Clinical immunology and the immunological basis of disease; Pharmacological interventions and new therapies. The manuscript management system is completely online and includes a very quick and fair peer-review system, which is all easy to use. Visit http://www.dovepress.com/testimonials.php to read real quotes from published authors. 